This item was submitted to Loughborough's Research Repository by the author.

Items in Figshare are protected by copyright, with all rights reserved, unless otherwise indicated.

\title{
Salivary IgA as a risk factor for upper respiratory infections in elite professional athletes
}

PLEASE CITE THE PUBLISHED VERSION

http://dx.doi.org/10.1249/MSS.0b013e31816be9c3

PUBLISHER

Lippincott, Williams \& Wilkins (c) American College of Sports Medicine

VERSION

AM (Accepted Manuscript)

LICENCE

CC BY-NC-ND 4.0

REPOSITORY RECORD

Neville, Vernon, Michael Gleeson, and Jonathan P. Folland. 2019. "Salivary Iga as a Risk Factor for Upper Respiratory Infections in Elite Professional Athletes”. figshare. https://hdl.handle.net/2134/10614. 
This item was submitted to Loughborough's Institutional Repository (https://dspace.lboro.ac.uk/) by the author and is made available under the following Creative Commons Licence conditions.

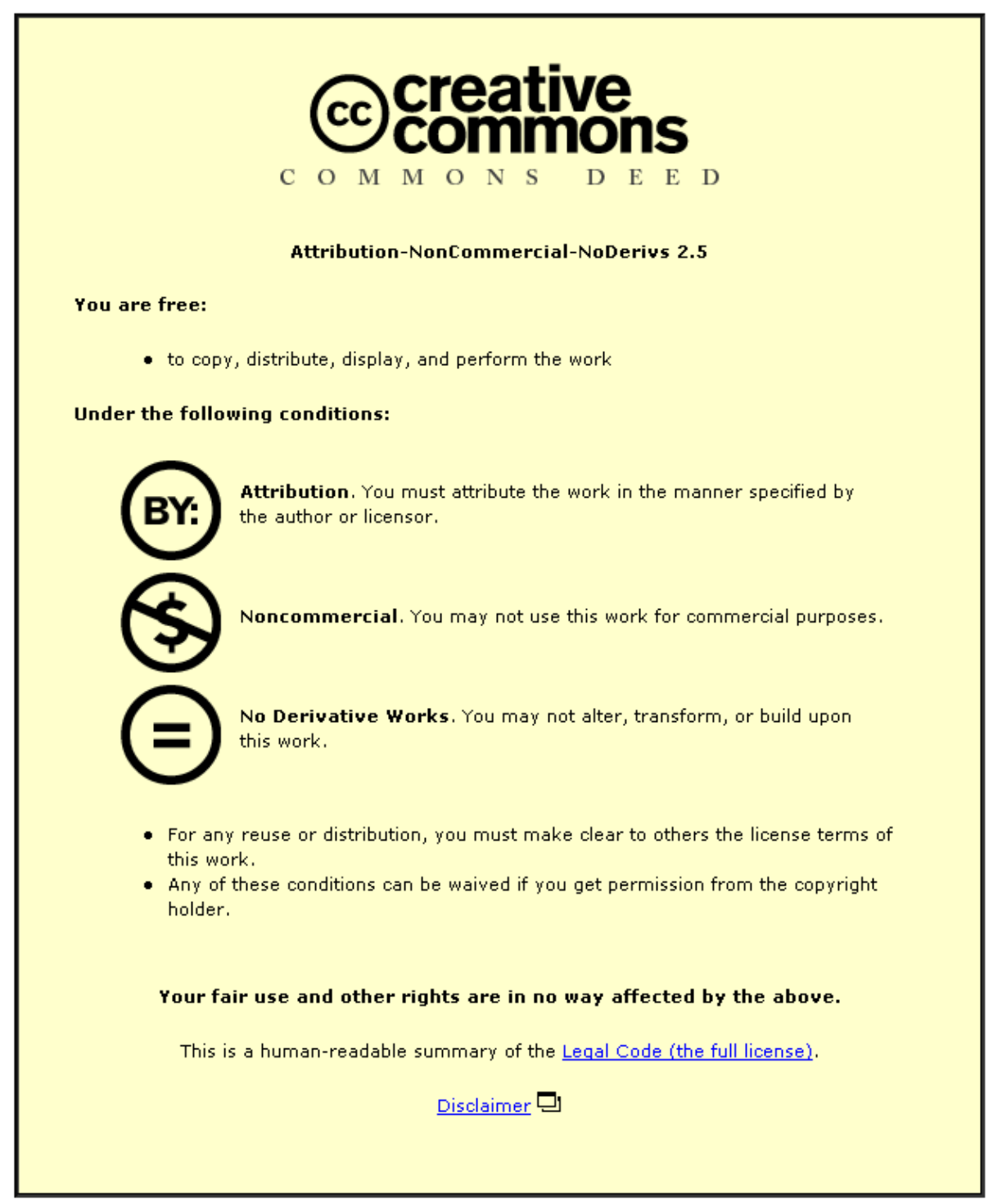

For the full text of this licence, please go to: http://creativecommons.org/licenses/by-nc-nd/2.5/ 
Neville, V., Gleeson, M. and Folland, J.P. (2008). Salivary IgA as a risk factor for upper respiratory infections in elite professional athletes. Medicine and Science in Sports and Exercise 40(7): 1228-1236.

Salivary IgA as a Risk Factor for Upper Respiratory Infections in Elite Professional Athletes

Authors:

Vernon Neville, Michael Gleeson and Jonathan P Folland

Affiliations:

School of Sport and Exercise Sciences, Loughborough University, Loughborough, United Kingdom.

Corresponding Author:

Name: $\quad$ Vernon Neville

Address: $\quad 5$ Castledine Street Extension, Loughborough, LE11 2NT, United Kingdom Email: $\quad$ vernon.neville@gmail.com

Tel: $\quad$ +44 (0) 7760258085

Fax: $\quad$ +44(0) 1509217182

Running Title:

S-IgA and Risk of URI in Athletes 


\section{ABSTRACT}

The relationship between physiological and psychological stress and immune function is widely recognised; however, there is little evidence to confirm a direct link between depressed immune function and incidence of illness in athletes. Purpose: To examine the relationship between salivary-Immunoglobulin $\mathrm{A}(\mathrm{s}-\mathrm{Ig} \mathrm{A})$ and upper respiratory infections (URI) in a cohort of professional athletes over a prolonged period of time. Methods: Thirtyeight elite America's Cup Yacht Racing athletes were studied over 50weeks of training. Resting, unstimulated saliva samples were collected weekly ( $38 \mathrm{~h}$ post-exercise, consistent time of day, fasted) together with clinically confirmed URI, training load and perceived fatigue rating. Results: S-IgA was highly variable within $(\mathrm{CV}: 48 \%)$ and between subjects (CV:71\%). No significant correlation was found between absolute s-IgA concentration and the incidence of URI among athletes $(r=0.11)$. However, a significant $(28 \%, \mathrm{P}<0.005)$ reduction in s-IgA occurred during the 3 weeks prior to URI episodes and returned to baseline by 2 weeks following URI. When an athlete did not have, or was not recovering from URI, a s-IgA value $<40 \%$ relative to their mean healthy s-IgA concentration indicated a one in two chance of contracting an URI within 3 weeks. Conclusion: On a group basis, relative s-IgA determined a substantial proportion of the variability in weekly URI incidence. The typical decline in an individual's relative s-IgA over the 3 weeks prior to URI appears to precede and contribute to URI risk, with the magnitude of the decrease related to the risk of URI, independent of the absolute s-IgA concentration. These findings have important implications for athletes and coaches in identifying periods of high URI risk.

Key Words: IMMUNOLOGY, ILLNESS, UNDERLYING FATIGUE, SAILING, AMERICA'S CUP 


\section{INTRODUCTION}

Paragraph 1 Upper respiratory infections (URI) are the most common medical complaint of athletes $(26,35)$ and can negatively affect training and performance (33). For example, during a two year training period prior to the $31^{\text {st }}$ America's Cup, $40 \%$ of all illnesses were URI and accounted for $60 \%$ of days absent from sailing due to illness (26). In addition, elite athletes seem to be more susceptible to URI than recreationally active or sedentary individuals (39), with the risk of illness increasing during periods of heavy training and competition $(21,30,32)$. This increased susceptibility to URI is thought to be largely due to a depression of immune system function as a result of multifactorial stress including physiological, psychological, environmental and behavioral $(4-6,42)$ (see Gleeson (11) for review).

Paragraph 2 Approximately 95\% of all infections are initiated at the mucosal surfaces (2), which are protected by antimicrobial proteins of which secretory immunoglobulin A (IgA) is the most abundant (3). Secretory $\operatorname{IgA}$ provides an immunological barrier by neutralizing and preventing viral pathogens from penetrating the body through the mucosal surfaces $(20,24)$. In the buccal cavity, the synthesis and secretion of salivary $\operatorname{IgA}(\mathrm{s}-\operatorname{Ig} \mathrm{A})$ responds almost instantaneously to stress (2), resulting in transitory fluctuations in concentration and secretion rate (40).

Paragraph 3 Elite athletes are frequently exposed to exercise stress, and the effects of both acute and chronic exercise on s-IgA have been well documented $(14,21,23,30,42)$ and appear to depend on the fitness level of the individual as well as the training load. In elite athletes, s-IgA concentration and secretion rate decrease after a bout of strenuous exercise, of either high volume (29) or maximal intensity (9), or during prolonged periods involving repeated bouts of strenuous training (21). Much of the immunology research in athletes has 
concentrated on post-exercise salivary immunity when athletes seem to experience a transitory decrease in s-IgA for up to $24 \mathrm{~h}$ post strenuous training or competition. It is during this "open window" period of immune depression (31) when athletes are thought to be at greatest risk of URI. However, there are few longitudinal studies that have examined the relationship between immune depression and the incidence of URI $(8,15,22,30)$ and these typically have had a low number of subjects or low sample collection frequency. Nevertheless, an absolute s-IgA concentration of less than $40 \mathrm{mg} \cdot \mathrm{L}^{-1}(15)$ and an absolute sIgA secretion rate of less than $40 \mu \mathrm{g} \cdot \min ^{-1}$ (8) have been reported to be associated with increased incidence of URI in athletes.

Paragraph 4 Large within and between subject variations in s-IgA concentration have been reported in elite rowers and swimmers, recreationally active and sedentary individuals $(10,25)$. This variation implies that the secretion of s-IgA may be specific to the individual and their recent environmental circumstances, and advocates regular monitoring of well controlled basal values to determine individual reference data. These variations also question the validity of studies which have few sample measures (13). Furthermore, consensus regarding the control of factors known to affect basal s-IgA has yet to be determined, such as: the residual effects of exercise, nutrition status (fasted vs non fasted), circadian rhythms and caffeine ingestion (see Gleeson et al. (16) for review). These inconsistencies in methodology have led to inconsistancies in the literature and make it difficult to compare studies (38). Furthermore, in determining URI, the majority of studies have used self-reporting illness logs that have not been validated against objective criteria (39) and may be prone to inconsistency and over-reporting (16). Few studies have used clinical diagnosis to confirm the presence of URI.

Paragraph 6 The overall aim of this study was to examine the relationship between s-IgA and URI in a relatively large cohort of athletes over a prolonged period of time (weekly 
samples for 50 weeks). The specific objectives were: to document the within and between athlete variability in resting s-IgA; examine the relationship between s-IgA and URI, and whether s-IgA values indicated the presence or imminent onset of URI; and to investigate the relationships of subjective fatigue rating and physical stress (sailing and training load) with s$\operatorname{IgA}$.

\section{METHODS}

Paragraph 7 Subjects. Thirty eight elite America's Cup Yacht Racing athletes (mean \pm SD: age $36 \pm 7$ years, body mass $92 \pm 12 \mathrm{~kg}$, body fat $14 \pm 4 \%$ and arm ergometer $\mathrm{VO}_{2} \mathrm{max}$ $50 \pm 5 \mathrm{ml} \cdot \mathrm{min}^{-1} \cdot \mathrm{kg}^{-1}$ ) were studied over 50 weeks of sailing and training. The subjects were all professional athletes contracted to one of the top four America's Cup teams, with their collective experience and success including: 20 Olympic Games representations, 8 Olympic medals, 90 World Championship titles and more than 100 America's Cup campaigns.

Paragraph 8 Experimental design. A prospective longitudinal study design was used to collect saliva samples, illness reports, training load and fatigue ratings over an 18-month sailing and training preparation period prior to the $32^{\text {nd }}$ America's Cup held in Valencia, Spain in 2007. Informed consent was obtained from all athletes and the study was conducted within the team's normal training and competition schedule and overseen by the team's sports science and medical support staff. The study was approved by the Loughborough University Ethical Advisory Committee.

Paragraph 9 America's Cup Yacht Racing. The America's Cup is the oldest trophy in modern day sports, dating back to 1851. It is regarded as the pinnacle of yacht racing and is held every three to four years. The 24-ton high performance racing yachts are sailed by 17 
athletes around a specific race course. Races are 1.5 to $2.5 \mathrm{~h}$ in duration and all maneuvers on-board are performed manually without assistance from stored energy. The physiological demands are specific to the role of the athlete and largely dependent on the weather conditions. The crew can be divided into two groups based on the physical demands of each position, with grinders, mastmen, bowmen and pitmen in high physically demanding roles, and helmsmen, navigators, trimmers, tacticians and strategists in moderately demanding roles.

Paragraph 10 Work load. The athletes' week typically consisted of 6 training days and one day of rest. Their working day was typically between 8 and $14 \mathrm{~h}$ in duration, beginning at 08:00 h with approximately $1 \mathrm{~h}$ of land-based strength and conditioning exercise ('training') followed by meetings and preparing the boats for sailing. The volume of sailing varied between 3 to $7 \mathrm{~h}$ per day whereafter, 1 to $3 \mathrm{~h}$ of boat maintenance was carried out. This was followed by further meetings and on some occasions an additional short bout of strength and conditioning exercise.

Paragraph 11 Sailing and Training data. In order to calculate an index of overall sailing and training load, the product of volume and intensity was ranked on a scale of 1 to 5 $(1=$ very low load; $5=$ very high load $)$ for sailing and training separately. These were combined for the cohort with a weighting of $2 / 3$ for sailing and $1 / 3$ for training to provide an index of combined sailing and training load.

Paragraph 12 Saliva collection. Saliva samples were collected weekly at 07:45 h in a fasted state, the day after a rest or no training day ensuring a minimum of $38 \mathrm{~h}$ rest after the previous training session. Whole mixed saliva samples were collected prior to training and breakfast or coffee (1) $5 \mathrm{~min}$ after consuming $250 \mathrm{ml}$ of water. Athletes sat quietly with their head tilted forward and passively dribbled (with minimal orofacial movement) approximately 
$1 \mathrm{ml}$ of unstimulated saliva into a pre-marked specimen container. Samples were placed in a pre-cooled insulated container and taken directly to the laboratory for analysis.

Paragraph 13 Saliva analysis. Saliva samples were analysed for s-IgA concentration within $2 \mathrm{~h}$ of collection. Salivary IgA concentration was determined by means of immunonephelometry using a BN ProSpec analyser (Dade-Behring Marburg GmbH, Marburg, Germany). In summary, IgA in human saliva forms immune complexes in an immunochemical reaction with specific antibodies. These complexes scatter a beam of light passed through the sample. The intensity of the scattered light is proportional to the concentration of IgA in the sample. The result is evaluated by comparison with a standard of known concentration. The reagents used were N Antiserum to Human IgA (Dade Behring Marburg GmbH, Marburg, Germany), produced by immunization of rabbits with highly purified human immunoglobulin A with Sodium azide $\left(<1\right.$ g. $\left.\mathrm{L}^{-1}\right)$ added as a preservative and N Diluent (Dade Behring Marburg GmbH, Marburg, Germany), which contains Phosphate buffered saline and sodium azide $\left(<1\right.$ g. $\left.\mathrm{L}^{-1}\right)$ as a preservative. Samples were assayed in duplicate after being brought to room temperature and spun at 14,000 rpm for 6 minutes. Supernatant was recovered and transferred to a sample cup. After a 1:5 dilution with NDiluent, sample and antiserum were incubated for 6 minutes prior to immunocomplexes measurement using a $380 \mathrm{~nm}$ light beam. The within-run coefficients of variation (CV) for each assay were on average $1.6 \%$ and the mean between-run $\mathrm{CV}$ was $3.7 \%$ with a total $\mathrm{CV}$ of 4\%. The limit of sensitivity was $14 \mathrm{mg} \cdot \mathrm{L}^{-1}$. Contaminated samples or those containing sputum were excluded from analysis.

Paragraph 14 Illness reports. Respiratory illness and infections were recorded by the team's Physician, who was present during all data collection and team training sessions. An URI was only recorded if the athlete required medication (either systemic or antibiotic) and missed at least one sailing or training session as a result of the illness (26). Medical 
consultations, allergies and the prescription of vitamin supplementation or prophylactic treatment were not considered an URI episode. A recurring illness was defined as "any URI occurring within one week of a previously recorded episode" and excluded from analysis.

Paragraph 15 Fatigue rating. A simple three scale subjective fatigue rating questionnaire was completed at the same time as saliva collection during the last 30 weeks of the study period. The questionnaire asked: "How rested do you feel?" to which there were three answers: "worse than normal", "normal" or "better than normal".

Paragraph 16 Statistical analysis. Data are expressed as mean \pm SEM, and the level of significance was set at $\mathrm{P}<0.05$. The reliability of s-IgA was calculated within and between subjects with the coefficient of variation (CV). Independent samples $t$ test was used to identify differences between athletes in high and moderate physically demanding roles. Pearson's product moment correlations were used to determine the strength of relationships.

Paragraph 17 The mean s-IgA concentration for each individual was calculated as the mean of all No URI values (i.e. s-IgA values were excluded from the mean when a URI episode was present), and individual relative s-IgA was calculated as percentage of this mean value. Paired samples $t$ test was used to assess any differences between s-IgA during URI and No URI. Relative s-IgA concentrations before, during and after URI were compared with repeated measures ANOVA and a Bonferroni post-hoc test to analyse where any differences lay. These procedures were also used to compare s-IgA for different ratings of fatigue. Analyses were performed using SPSS version 14.0 for Windows.

Paragraph 18 The incidence of low s-IgA values $(<40 \%$ and $<70 \%$ of an individual's mean) was calculated in the weeks before, during and after URI. The probability of low s-IgA leading to URI within 3 weeks (Predictability of URI), when URI was not present or recent (i.e. excluding during URI or 1-week post-URI) was calculated as the number of samples during pre-URI as a percentage of pre- and No URI. 


\section{RESULTS}

Paragraph 19 Over the 50-week study period 1,424 saliva samples were analysed, with a mean s-IgA concentration of $136 \pm 3 \mathrm{mg} \cdot \mathrm{L}^{-1}$. Salivary IgA concentration was highly variable within-subjects, with a mean coefficient of variation $(\mathrm{CV})$ of $48 \%$. The difference in the mean value between the lowest and the highest individual was almost 10 fold $\left(35 \pm 4 \mathrm{mg} \cdot \mathrm{L}^{-1}\right.$ vs. $314 \pm 27 \mathrm{mg} \cdot \mathrm{L}^{-1}$; Figure $\left.1 \mathrm{~A}\right)$ and the between-subjects $\mathrm{CV}$ was $71 \%$. The s-IgA concentration of athletes with sailing roles of moderate and high physical demands were similar (moderate: $149 \pm 20 \mathrm{mg} \cdot \mathrm{L}^{-1}$; high: $127 \pm 12 \mathrm{mg} \cdot \mathrm{L}^{-1}$ ).

Paragraph 20 A total of 102 incidents of URI were recorded, resulting in 129 weeks of infection with symptoms ranging from 1 to 3 weeks in duration. The incidence of URI was on average $2.7 \pm 0.3$ infections per athlete over the 50-week period (Figure 1B), and was similar for athletes in roles with moderate $(2.9 \pm 0.5)$ and high $(2.5 \pm 0.4)$ physical demands.

Paragraph 21 There was no relationship between an athlete's mean s-IgA and his number of URI ( $r=0.11)$. When s-IgA values were normalised to each individual's mean, relative s-IgA concentration was $28 \%$ lower during URI than when there was no URI $(\mathrm{P}<$ 0.005; Figure 2). For the cohort, the number of URI in each week was inversely related to the mean weekly relative s-IgA concentration (Figure 3). The four lowest weekly mean relative s-IgA values $(<70 \%)$ were recorded during the pre-season training period (March and April) and three of these weeks were coincident with the highest incidence of URI. Relative s-IgA declined progressively during the 3 weeks prior to URI, being significantly lower during URI in comparison to 4 weeks prior, before returning to above baseline by 2 weeks following URI (Figure 4). 
Paragraph 22 The frequency of low s-IgA values $(<40 \%$ of an individual's mean healthy value) was higher in the weeks prior to, during and immediately after URI, than when no URI was present or imminent, being almost 6-fold greater in the week before infection (Table 1). When an individual did not have an URI or was not recovering from an URI, a low relative s-IgA value $(<40 \%)$ suggested a $48 \%$ chance $(23 / 48)$ of contracting an URI within 3 weeks, compared with a $28 \%$ chance $(74 / 263)$ of URI for relative s-IgA values of less than $70 \%$. However, during the 3 weeks prior to URI, $89 \%$ and $65 \%$ of s-IgA values were greater than $40 \%$ and $70 \%$ of relative s-IgA, respectively.

Paragraph 23 The mean total sailing and training exposure for each athlete over the 50 weeks was $986 \mathrm{~h}$, (749 h sailing; $237 \mathrm{~h}$ training) and the mean weekly combined sailing and training load ranged from 2.0 to 4.4. No relationship was found between weekly combined sailing and training load and URI $(r=0.002)$. However, a significant correlation was found between the weekly sailing and training load and the weekly percentage of relative mean sIgA concentration $(r=0.41$, Figure 5$)$.

Paragraph 24 There was a difference between the athletes' relative s-IgA concentration according to their fatigue rating, with s-IgA being significantly different for each of the fatigue ratings: "better than normal" $131 \pm 8 \%$; "normal" $103 \pm 4 \%$; "worse than normal" 69 $\pm 5 \%(\mathrm{P}<0.005$, Figure 6$)$.

\section{DISCUSSION}

Paragraph 25 This is the largest salivary immunology study on elite athletes to date. The main findings suggest that the relative s-IgA concentration is associated with, and can help to predict URI in elite athletes. For the cohort, relative s-IgA determined a substantial 
proportion of the variability of URI incidence. The typical decline in an individual's relative s-IgA over the 3 weeks before URI appears to pre-empt and contribute to URI risk, with the level of risk related to the extent of the decline in s-IgA, independent of the absolute concentration. In addition, the s-IgA of elite athletes is highly variable, both within and between subjects, and is related to the athlete's perception of underlying fatigue. These results suggest that regular monitoring of resting s-IgA may benefit athletes and coaches in determining the risk of URI and fatigue in elite athletes.

Paragraph 26 The mean s-IgA concentration in the current study is similar to that reported by several previous studies in exercise immunology $(17,37,41)$. However, the differences between studies are extensive, with some studies having a 15-fold greater (28) mean s-IgA concentration than others (23). These differences could be attributed to variations in: methodology, assay assessment techniques, control for basal resting values, different cohorts of subjects and large between-subject variability. It is therefore difficult to make comparisons between studies (38), particularly with respect to absolute values of s-IgA.

Paragraph 27 The volume of sailing per week was greater (almost 30\%) than that reported during the $31^{\text {st }}$ America's Cup (26), as was the ratio of sailing to training volume (26). This was possibly due to the more favourable sailing conditions at the venue of the $32^{\text {nd }}$ America's Cup. The correlation between the combined sailing and training load and the athletes' weekly s-IgA was in accordance with previous reports $(8,21,28-30,42)$.

Paragraph 28 The incidence of URI (2.7 episodes per 1,000 h sailing and training) was similar to that previously reported in America's Cup yacht racing (26), with a similar incidence for athletes in sailing roles with high and moderate physical demands.

Paragraph 29 The large within-subject variation in the current study (CV: 48\%) concurs with results found in elite level rowing (25) and swimming (10). Elite athletes have been reported to have greater within-subject variability than recreationally active or sedentary 
individuals (10), which may be due to the many stressors encountered as a result of high training loads and competition. These variations may be characteristic of the individual and their response to recent circumstances, and may indicate that some athletes are more susceptible (or adaptable) to stress than others. This variation was despite carefully controlling for a range of factors known to influence s-IgA. In fact, the methodological care with which samples were collected in standardised conditions (after a rest day, at a consistent time of day, in a fasted state and without caffeine ingestion) was one of the strengths of the current study. Many studies have not controlled for factors known to influence the concentration of s-IgA such as: the residual effects of exercise (10), large data collection windows $(10,36)$ and caffeine ingestion $(15,30)$. Other factors known to influence s-IgA which have not been controlled for in the literature include: time of day and circadian rhythm effects (6) and nutritional status (12). Whilst many of these inconsistencies may explain some of the discrepancies found in the literature, even when well controlled for in this study, there was still a large within-subject variability, which highlights the complex nature of the mucosal immune system. Saliva samples were collected $38 \mathrm{~h}$ post-exercise to ensure that the athletes were well rested and exclude any residual effects of exercise; hence, these results are related to the resting status of the individual and should not be compared with the effects of acute stress or temporal changes in s-IgA.

Paragraph 30 Another factor affecting baseline values is sample size (18). The current study collected up to 50 samples for each athlete, which is considerably more than the range of 2 to 13 samples in most previous longitudinal studies $(8,10,13-15,19,21-23,34,41)$. With s-IgA being highly variable, the smaller the number of samples, the less likelihood of detecting a real change (18), which could account for some of the inconsistencies in the literature. In addition, a low $N$ means that any outlier values may have a large influence on 
the correlation coefficient, thereby increasing the risk or chance effect of a significant correlation.

Paragraph 31 The large between-subjects variability in s-IgA concentration found in this study $(\mathrm{CV}: 71 \%)$ concurs with previous reports $(10,25)$ and strongly indicates that resting values of s-IgA are specific to the individual. Hence, it is the aetiology of this variability which may provide important answers to athletes' susceptibility to illness.

Paragraph 32 No association was found between the absolute mean s-IgA concentration of each athlete and the incidence of URI, indicating that athletes with low s-IgA concentration were no more at risk of URI than athletes with high values. This is contrary to previous reports, which have suggested that an absolute concentration of $<40 \mathrm{mg} \cdot \mathrm{L}^{-1}$ (15) or an absolute rate of secretion of $<40 \mu \mathrm{g} \cdot \mathrm{min}^{-1}$ (8) may increase the risk of URI. Based on these previous reports, the athlete with the lowest mean s-IgA concentration $\left(35 \pm 4 \mathrm{mg} \cdot \mathrm{L}^{-1}\right)$ in the current study would be at chronic risk of infection, when in fact this athlete reported no incidence of illness during the study, and coincidently, the athlete with the highest mean value $\left(314 \pm 27 \mathrm{mg} \cdot \mathrm{L}^{-1}\right)$ reported the greatest number of URI (8). Based on these data, relative values are the preferable means of expressing resting s-IgA and provided sufficient resting samples are measured, will provide a valid baseline for each individual.

Paragraph 33 A main finding of this study was the association between s-IgA and URI, where the weekly mean relative s-IgA concentration was negatively related to the incidence of URI $(r=-0.54, \mathrm{P}<0.005)$. This indicates that on a group basis the weekly mean s-IgA determines a substantial proportion (29\%) of the variation in URI incidence. Hence, monitoring group s-IgA may assist coaching staff in identifying periods of high risk in order to apply appropriate intervention (see Pyne et al. (34) for a review of intervention strategies). Previously, the relationship between s-IgA and URI in athletes has been less than convincing, with only a few studies having suggested an association $(8,13,15,22)$. These results are 
therefore important in confirming that s-IgA plays an important role in the incidence of URI and that the changes in s-IgA within an individual may be directly responsible for URI risk or may be a surrogate measure for some other immune system function. Interestingly, the lowest weekly s-IgA values for the group occurred during the first 6 weeks of training after a 2month winter off-season period, and coincided with the highest weekly incidence of URI. This is likely to be a combined result of: changes in environment, increased sailing and training load, changes in diet, psychological stress associated with returning to the competitive team environment and exposure to pathogens during public travel on return to the team (34).

Paragraph 34 On an individual basis there was also a difference in s-IgA concentration ( 30\%) between when an athlete had URI and when No URI was present. When the time course of s-IgA was examined in the weeks before, during and after an URI episode, there was a progressive decline in the 3-weeks prior to URI and a subsequent return to baseline within 2-weeks following URI. The cause and effect relationship as inferred by Mackinnon et al. (22), where the decrease in s-IgA prior to infection could be the result of the incubation period prior to expression of URI symptoms, is unlikely to apply to the findings of the present study, as the incubation period of URI is usually only 1 to 3 days (7). Therefore, it is postulated that the reduction in s-IgA in the weeks prior to URI is a contributing factor to the subsequent incidence of URI. It seems that on average, a $30 \%$ reduction in relative s-IgA from healthy values may increase the risk of URI, which is further supported by the increased frequency (2.5-fold) of s-IgA values less than $70 \%$ of an individual's mean healthy value during the week prior to URI when compared with times when no infection was present. The results further suggest that the greater the drop below an individual's mean healthy s-IgA concentration, the greater the risk of URI. 
Paragraph 35 An important finding of this study was that for individual athletes low relative s-IgA concentrations were associated with an increased risk of URI. For example: when an athlete was healthy (did not have URI or was not recovering from URI), a low s-IgA value $(<40 \%$ of an individual's mean healthy value) suggested a $48 \%$ chance of contracting an URI within 3 weeks, compared with a $28 \%$ chance when values were below $70 \%$ of an individual's healthy mean s-IgA concentration. Therefore, the lower the s-IgA value below baseline, the greater the probability of contracting an URI. Although it should be noted that $38 \%$ of URI were not preceded by values below baseline and only $11 \%$ of URI were preceded by values $<40 \%$. Hence, the absence of low s-IgA values was no guarantee of remaining healthy, indicating the multifactorial nature of immunity, and that factors other than reduced s-IgA alone contribute to the risk of infection.

Paragraph 36 No significant relationship was found between sailing and training load and the incidence of URI, which is contrary to previous reports in a number of different sports, including: elite level swimming (39), elite tennis (30) and endurance running $(27,32)$. This may be due to the difficulty in accurately determining the total work load that each individual is exposed to, as athletes are often required to perform large volumes of work over and above the physical requirements of sailing and training, including: boat maintenance, sail packing, boat sanding, as well as the psychological stress of design and performance meetings .

Paragraph 37 As fatigue is common in athletes during periods of heavy training and competition, the relationship between salivary immunity and recovery is of great interest. To our knowledge, this is the first report of subjective fatigue being associated with relative sIgA concentration. The results suggest that a simple fatigue rating reflects immune status to some extent, and appears to validate the use of a simple subjective questionnaire in monitoring underlying fatigue and recovery of athletes. These findings also imply that an 
athlete's underlying fatigue or psychological state may have a major influence on s-IgA in addition to the combined sailing and training load.

Paragraph 38 In summary, the results of this study confirm the role of s-IgA in the incidence of URI in elite athletes. The weekly mean relative s-IgA concentration for the cohort was negatively related to the incidence of URI, indicating that on a group basis the weekly mean s-IgA determines a substantial proportion of the variation in URI incidence. No association was found between the absolute mean s-IgA concentration of each athlete and the incidence of URI, indicating that athletes with low mean s-IgA concentration were at no greater risk of infection. Consequently, relative values are the preferred means of expressing basal s-IgA concentration. The large within and between subjects variability strongly indicates that basal values of s-IgA are specific to the individual and a relatively large number of samples are required to determine baseline values. Elucidating the aetiology of this variability would enhance our understanding of athletes' susceptibility to illness. The reduction in s-IgA in the weeks prior to URI appears to be a contributing factor to the subsequent incidence of URI, with the magnitude of the decrease related to the risk of URI. Furthermore, a simple fatigue rating appears to reflect changes in salivary immunity. The results presented in this study advocate frequent monitoring of well controlled resting s-IgA in elite athletes. If the results are rapidly available, they may assist athletes and their support staff in identifying periods of high URI risk so that appropriate preventative strategies can be applied. Furthermore, the use of a simple fatigue questionnaire can provide coaches with valuable information on the underlying fatigue status of the athlete.

\section{Acknowledgements:}


We wish to thank Dr Cristina Perez Encinas and Dr Enrique Rodilla Sala for the clinical diagnosis and collection of URI data and Mr Sergio Rada Ruiz for his assistance in the collection of saliva samples. We are also grateful to Dr Alfredo Colomer and Dr Alfredo Montoro Soriano from Laboratorio Montoro, Valencia, Spain for their assistance in the collection and analysis of saliva samples. Our appreciation is also extended to the America's Cup athletes who took part in this study.

The results of this study do not necessarily constitute endorsement by ACSM.

\section{Contributors:}

All authors participated in the study design, data interpretation and writing of this manuscript. VN was the principal investigator and data collector.

\section{Conflict of Interest statement:}

The authors are not aware of any conflict of interest.

\section{REFERENCES}

1. Bishop NC, Walker GJ, Scanlon GA, Richards S, Rogers E. Salivary IgA responses to prolonged intensive exercise following caffeine ingestion. Med Sci Sports Exerc. 2006; 38 (3): 513-9. 
2. Bosch JA, Ring C, de Geus EJ, Veerman EC, Amerongen AV. Stress and secretory immunity. Int Rev Neurobiol. 2002; 52: 213-53.

3. Brandtzaeg P. Role of secretory antibodies in the defence against infections. Int J Med Microbiol. 2003; 293 (1): 3-15.

4. Calder PC, Jackson AA. Undernutrition, Infection and Immune Function. Nutrition Research Reviews. 2000; 13: 3-29.

5. Cohen S, Doyle WJ, Skoner DP. Psychological stress, cytokine production, and severity of upper respiratory illness. Psychosom Med. 1999; 61 (2): 175-80.

6. Dawes C. Circadian rhythms in human salivary flow rate and composition. $J$ Physiol. 1972; 220 (3): 529-45.

7. Department of Health CDCB. You've got what? : prevention and control of notifiable and other infectious diseases in children and adults. South Australia: Government of South Australia, 2005: 48-49.

8. Fahlman MM, Engels HJ. Mucosal IgA and URTI in American college football players: a year longitudinal study. Med Sci Sports Exerc. 2005; 37 (3): 374-80.

9. Fahlman MM, Engels HJ, Morgan AL, Kolokouri I. Mucosal IgA response to repeated wingate tests in females. Int J Sports Med. 2001; 22 (2): 127-31.

10. Francis JL, Gleeson M, Pyne DB, Callister R, Clancy RL. Variation of salivary immunoglobulins in exercising and sedentary populations. Med Sci Sports Exerc. 2005; 37 (4): 571-8.

11. Gleeson M. Immune function in sport and exercise. J Appl Physiol. 2007; 103 (2): 693-9.

12. Gleeson M, Bishop NC. Elite athlete immunology: importance of nutrition. Int $J$ Sports Med. 2000; 21 Suppl 1: S44-50. 
13. Gleeson M, Hall ST, McDonald WA, Flanagan AJ, Clancy RL. Salivary IgA subclasses and infection risk in elite swimmers. Immunol Cell Biol. 1999; 77 (4): 3515.

14. Gleeson M, McDonald WA, Pyne DB, Clancy RL, Cripps AW, Francis JL, Fricker PA. Immune status and respiratory illness for elite swimmers during a 12-week training cycle. Int J Sports Med. 2000; 21 (4): 302-7.

15. Gleeson M, McDonald WA, Pyne DB, Cripps AW, Francis JL, Fricker PA, Clancy RL. Salivary IgA levels and infection risk in elite swimmers. Med Sci Sports Exerc. 1999; 31 (1): 67-73.

16. Gleeson M, Pyne DB, Callister R. The missing links in exercise effects on mucosal immunity. Exerc Immunol Rev. 2004; 10: 107-28.

17. Halson SL, Lancaster GI, Jeukendrup AE, Gleeson M. Immunological responses to overreaching in cyclists. Med Sci Sports Exerc. 2003; 35 (5): 854-61.

18. Hopkins WG. Measures of reliability in sports medicine and science. Sports Med. 2000; $30(1): 1-15$.

19. Klentrou P, Cieslak T, MacNeil M, Vintinner A, Plyley M. Effect of moderate exercise on salivary immunoglobulin A and infection risk in humans. Eur J Appl Physiol. 2002; 87 (2): 153-8.

20. Lamm ME. Interaction of antigens and antibodies at mucosal surfaces. Annu Rev Microbiol. 1997; 51: 311-40.

21. Libicz S, Mercier B, Bigou N, Le Gallais D, Castex F. Salivary IgA response of triathletes participating in the French Iron Tour. Int J Sports Med. 2006; 27 (5): 38994. 
22. Mackinnon L, Ginn E, Seymour GJ. Temporal relationship between decreased Salivary $\operatorname{IgA}$ and upper respiratory tract infection in elite athletes. Aus J Sci Med Sport. 1993; 25 (4): 94-99.

23. Mackinnon LT, Ginn E, Seymour GJ. Decreased salivary immunoglobulin A secretion rate after intense interval exercise in elite kayakers. Eur J Appl Physiol Occup Physiol. 1993; 67 (2): 180-4.

24. Mazanec MB, Nedrud JG, Kaetzel CS, Lamm ME. A three-tiered view of the role of IgA in mucosal defense. Immunol Today. 1993; 14 (9): 430-5.

25. Nehlsen-Cannarella SL, Nieman DC, Fagoaga OR, Kelln WJ, Henson DA, Shannon M, Davis JM. Saliva immunoglobulins in elite women rowers. Eur J Appl Physiol. 2000; 81 (3): 222-8.

26. Neville VJ, Molloy J, Brooks JH, Speedy DB, Atkinson G. Epidemiology of injuries and illnesses in America's Cup yacht racing. Br J Sports Med. 2006; 40 (4): 304-11; discussion 311-2.

27. Nieman DC. Is infection risk linked to exercise workload? Med Sci Sports Exerc. 2000; 32 (7 Suppl): S406-11.

28. Nieman DC, Henson DA, Dumke CL, Lind RH, Shooter LR, Gross SJ. Relationship between salivary IgA secretion and upper respiratory tract infection following a 160km race. J Sports Med Phys Fitness. 2006; 46 (1): 158-62.

29. Nieman DC, Henson DA, Fagoaga OR, Utter AC, Vinci DM, Davis JM, NehlsenCannarella SL. Change in salivary IgA following a competitive marathon race. Int $J$ Sports Med. 2002; 23 (1): 69-75.

30. Novas AM, Rowbottom DG, Jenkins DG. Tennis, incidence of URTI and salivary IgA. Int J Sports Med. 2003; 24 (3): 223-9. 
31. Pedersen BK, Kappel M, Klokker M, Nielsen HB, Secher NH. The immune system during exposure to extreme physiologic conditions. Int J Sports Med. 1994; 15 Suppl 3: S116-21.

32. Peters EM, Bateman ED. Ultramarathon running and upper respiratory tract infections. An epidemiological survey. S Afr Med J. 1983; 64 (15): 582-4.

33. Pyne DB, Gleeson M. Effects of intensive exercise training on immunity in athletes. Int J Sports Med. 1998; 19 Suppl 3: S183-91; discussion S191-4.

34. Pyne DB, Gleeson M, McDonald WA, Clancy RL, Perry C, Jr., Fricker PA. Training strategies to maintain immunocompetence in athletes. Int J Sports Med. 2000; 21 Suppl 1: S51-60.

35. Robinson D, Milne C. Medicine at the 2000 Sydney Olympic Games: the New Zealand health team. Br J Sports Med. 2002; 36 (3): 229.

36. Sari-Sarraf V, Reilly T, Doran D, Atkinson G. Effects of Repeated Bouts of SoccerSpecific Intermittent Exercise on Salivary IgA. Int J Sports Med. 2007.

37. Sari-Sarraf V, Reilly T, Doran DA. Salivary IgA response to intermittent and continuous exercise. Int J Sports Med. 2006; 27 (11): 849-55.

38. Shephard RJ. Special feature for the Olympics: effects of exercise on the immune system: overview of the epidemiology of exercise immunology. Immunol Cell Biol. 2000; 78 (5): 485-95.

39. Spence L, Brown WJ, Pyne DB, Nissen MD, Sloots TP, McCormack JG, Locke AS, Fricker PA. Incidence, etiology, and symptomatology of upper respiratory illness in elite athletes. Med Sci Sports Exerc. 2007; 39 (4): 577-86.

40. Stone AA, Cox DS, Valdimarsdottir H, Neale JM. Secretory IgA as a measure of immunocompetence. J Human Stress. 1987; 13 (3): 136-40. 
41. Tharp GD. Basketball exercise and secretory immunoglobulin A. Eur J Appl Physiol Occup Physiol. 1991; 63 (3-4): 312-4.

42. Tomasi TB, Trudeau FB, Czerwinski D, Erredge S. Immune parameters in athletes before and after strenuous exercise. J Clin Immunol. 1982; 2 (3): 173-8. 


\section{FIGURE LEGENDS}

FIGURE 1 - Saliva IgA concentration (A, mean \pm range) and the number of Upper Respiratory Infections (B) for each athlete over the 50 weeks

FIGURE 2 - Saliva IgA concentration during URI (72 $\pm 5 \%)$ and No URI (100 $\pm 0 \%)$.

Data are mean \pm SEM of 31 athletes that reported an infection, with each individual's relative S-IgA values averaged for URI or not. * $\mathrm{P}<0.005$

FIGURE 3 - Scatter plot of the weekly number of URI within the subject cohort and Salivary $\operatorname{IgA}$ (mean of relative values for each individual) $\left(r=0.54, r^{2}=0.29, N=50, \mathrm{P}<0.005\right)$.

FIGURE 4 - Salivary IgA concentration for each week Pre, during and Post all infections ( $N$ $=102$ ). Data are mean \pm SEM of individual relative S-IgA (percentage of No URI mean values). * URI significantly different to -4 weeks, +1 week, +2 weeks, $\mathrm{P}<0.005 ; * *-1$ week significantly different to +2 weeks, $\mathrm{P}<0.005$.

FIGURE 5 - Scatter plot of the weekly Salivary IgA, mean relative values, and the combined Sailing and Training load $\left(r=0.41, r^{2}=0.17, N=50, \mathrm{P}<0.005\right)$.

FIGURE 6 - Salivary IgA concentration for different fatigue ratings. Data are Mean \pm SEM of 38 athletes, with each individual's relative S-IgA values averaged for each rating. * S-IgA for each rating significantly different to the other two $(N=38, \mathrm{P}<0.005)$. 


\section{TABLES}

TABLE 1. Number and incidence of low relative salivary IgA values $(<70 \%$ and $<40 \%$ of an individual's healthy average) before, during and after URI.

\begin{tabular}{lccc}
\hline & $\begin{array}{c}\text { Total number of } \\
\text { s-lgA samples }\end{array}$ & $\begin{array}{c}\text { Number (\% incidence) } \\
\text { of s-IgA }<70 \%\end{array}$ & $\begin{array}{c}\text { Number (\% incidence) } \\
\text { of s-IgA }<40 \%\end{array}$ \\
\hline No URI & 1020 & $189(18.5)$ & $25(2.5)$ \\
\hline 3weeks pre-URI & 56 & $12(21.4)$ & $3(5.4)$ \\
2weeks pre-URI & 71 & $25(35.2)$ & $12(11.3)$ \\
1week pre-URI & 83 & $37(44.6)$ & $15(13.8)$ \\
\hline During URI & 109 & $52(47.7)$ & $10(11.8)$ \\
1week post-URI & 85 & $21(24.7)$ & $\mathbf{4 8}$ \\
\hline Predictability of URI ${ }^{*}(\%)$ & & $\mathbf{2 8}$ & \\
\hline
\end{tabular}

"The Predictability of URI was calculated as the percentage of values below each threshold that led to URI within 3 weeks, when URI was not present or recent (i.e.: excluding during URI and 1 week post-URI). 
A

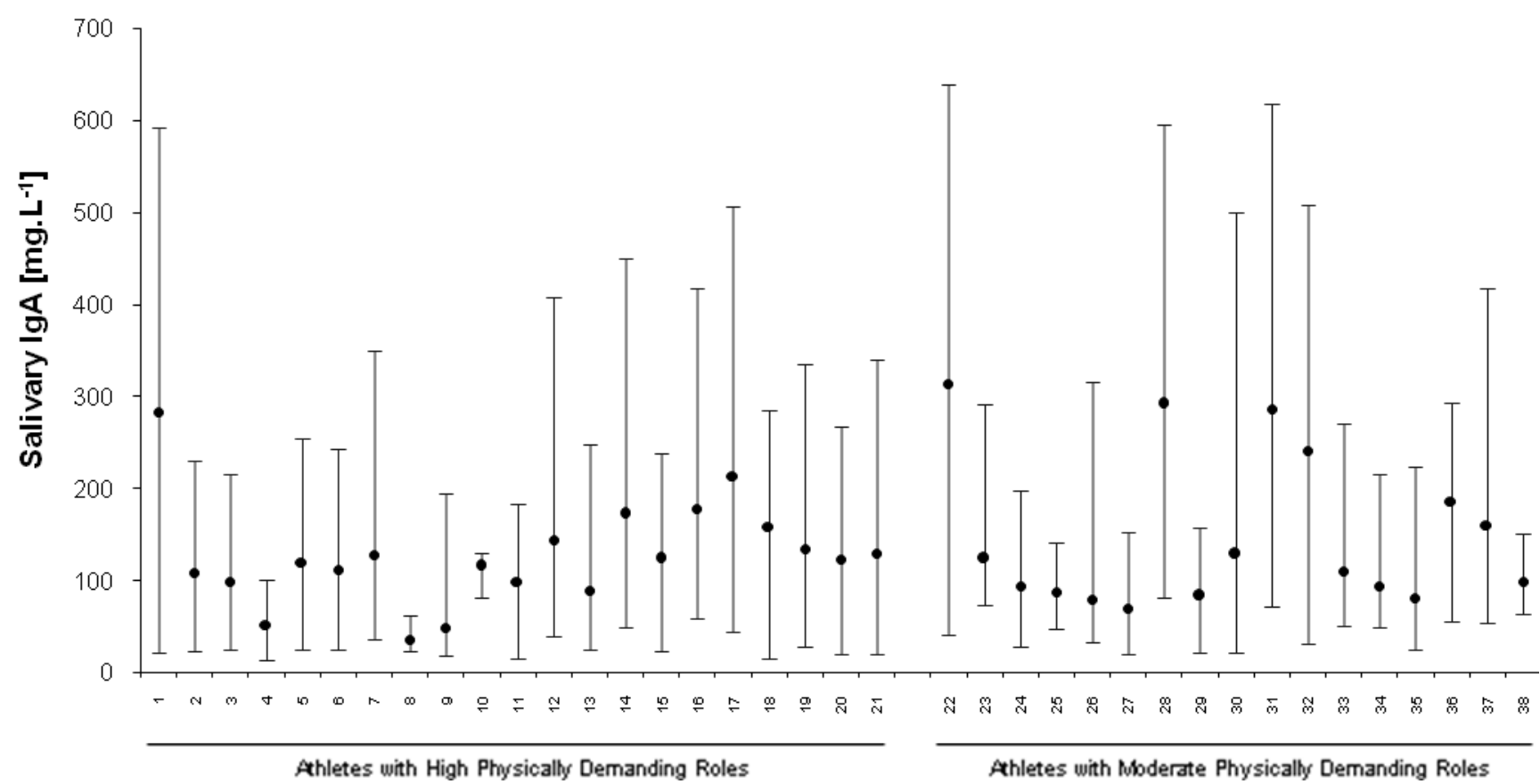

B

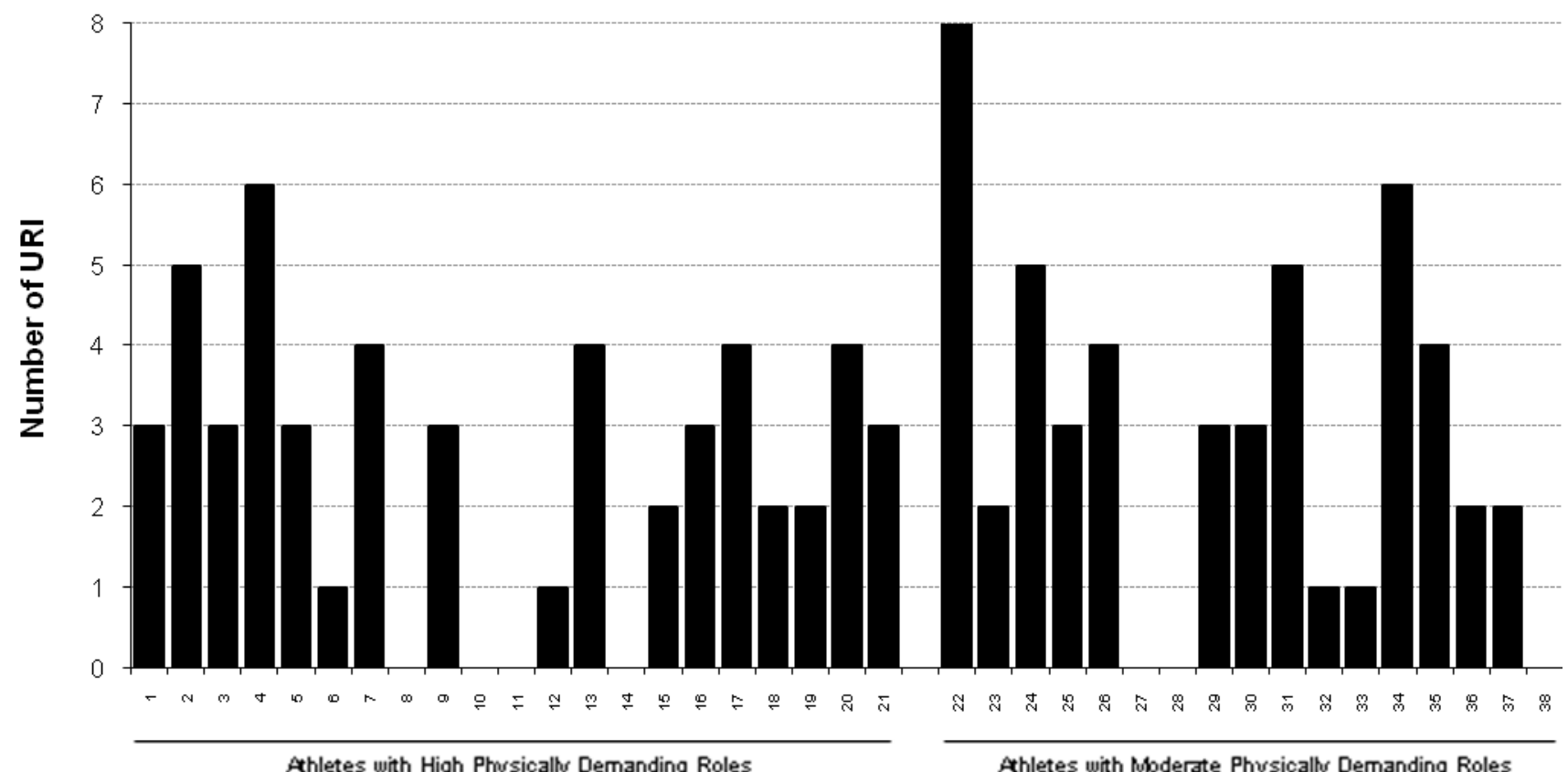

Figure 1: Saliva IgA concentration (A, mean \pm range) and the number of Upper Respiratory Infections (B) for each athlete over the 50 weeks 


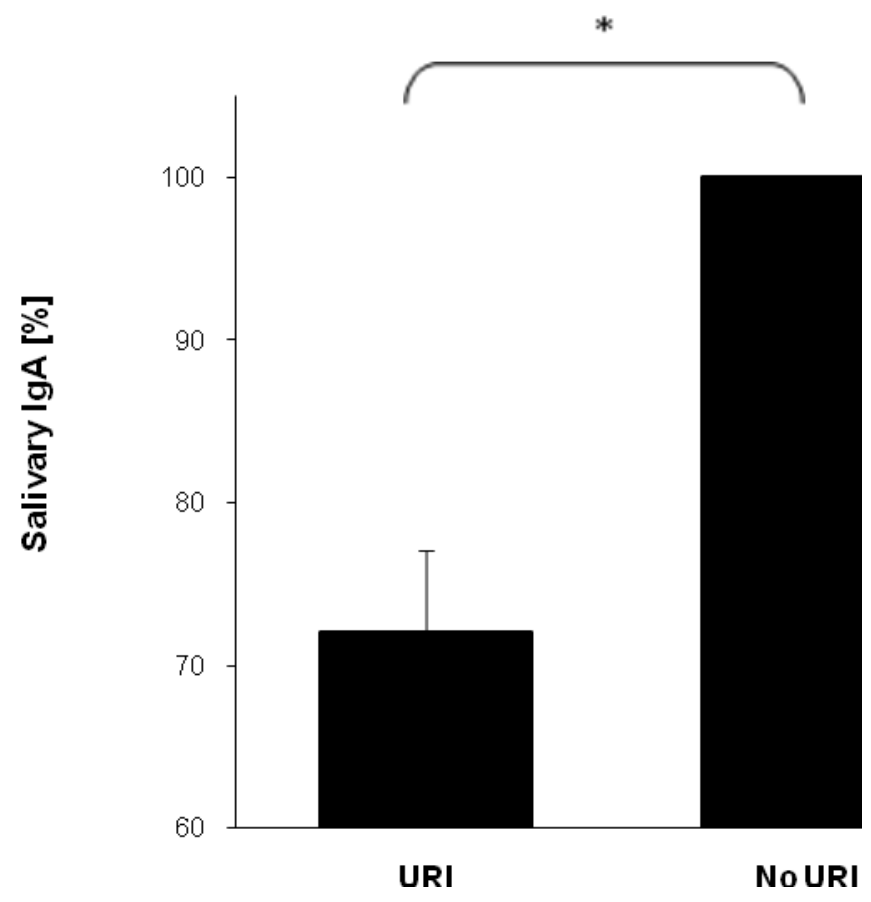

Figure 2: Saliva IgA concentration during URI $(72 \pm 5 \%)$ and No URI $(100 \pm 0 \%)$. Data are mean \pm SEM of 31 athletes that reported an infection, with each individual's relative $\mathrm{S}-\mathrm{IgA}$ values averaged for URI or not. * $\mathrm{P}<0.005$. 


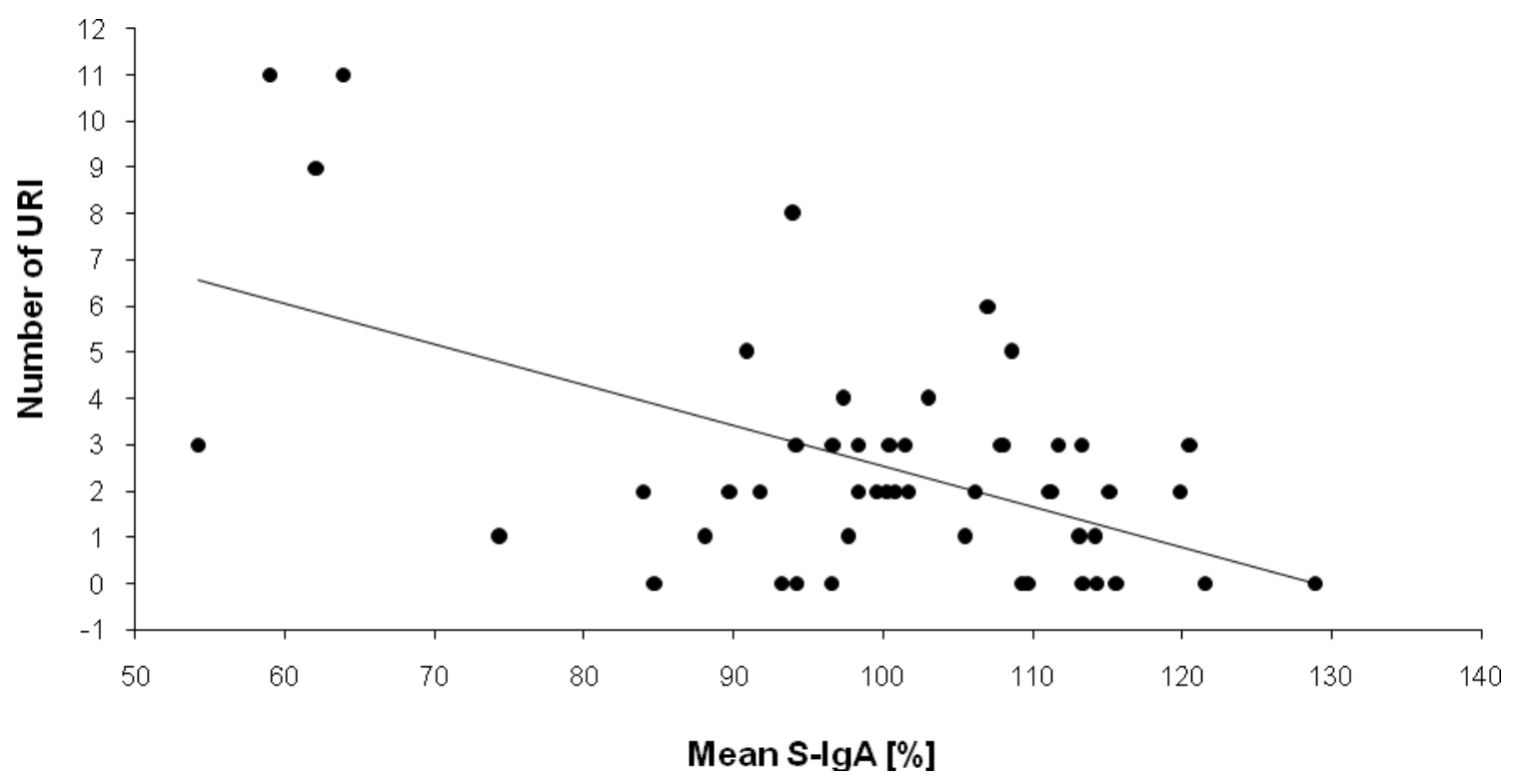

Figure 3: Scatter plot of the weekly number of URI within the subject cohort and

Salivary $\operatorname{IgA}$ (mean of relative values for each individual) $\left(r=0.54, r^{2}=0.29, N=50\right.$, $\mathrm{P}<0.005)$. 


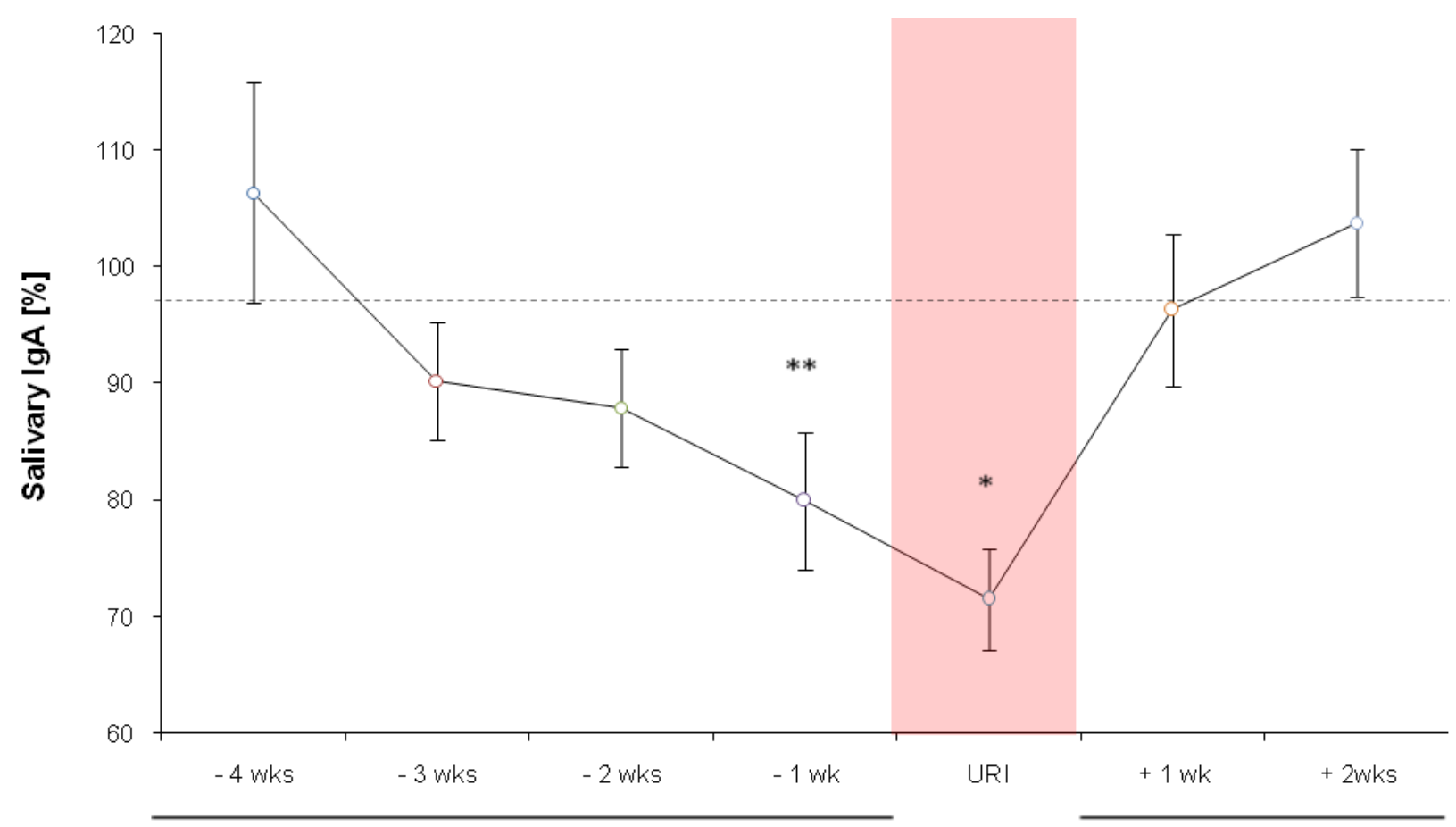

Pre Infection

Post Imfection

Figure 4: Salivary IgA concentration for each week Pre, during and Post all infections $(N=$ 102). Data are mean \pm SEM of individual relative $S$ - IgA (percentage of No URI mean values). $*$ URI significantly different to $-4 \mathrm{wks},+1 \mathrm{wk},+2 \mathrm{wks}, \mathrm{P}<0.005$; ** $-1 \mathrm{wk}$ significantly different to +2 wks, $\mathrm{P}<0.005$. 


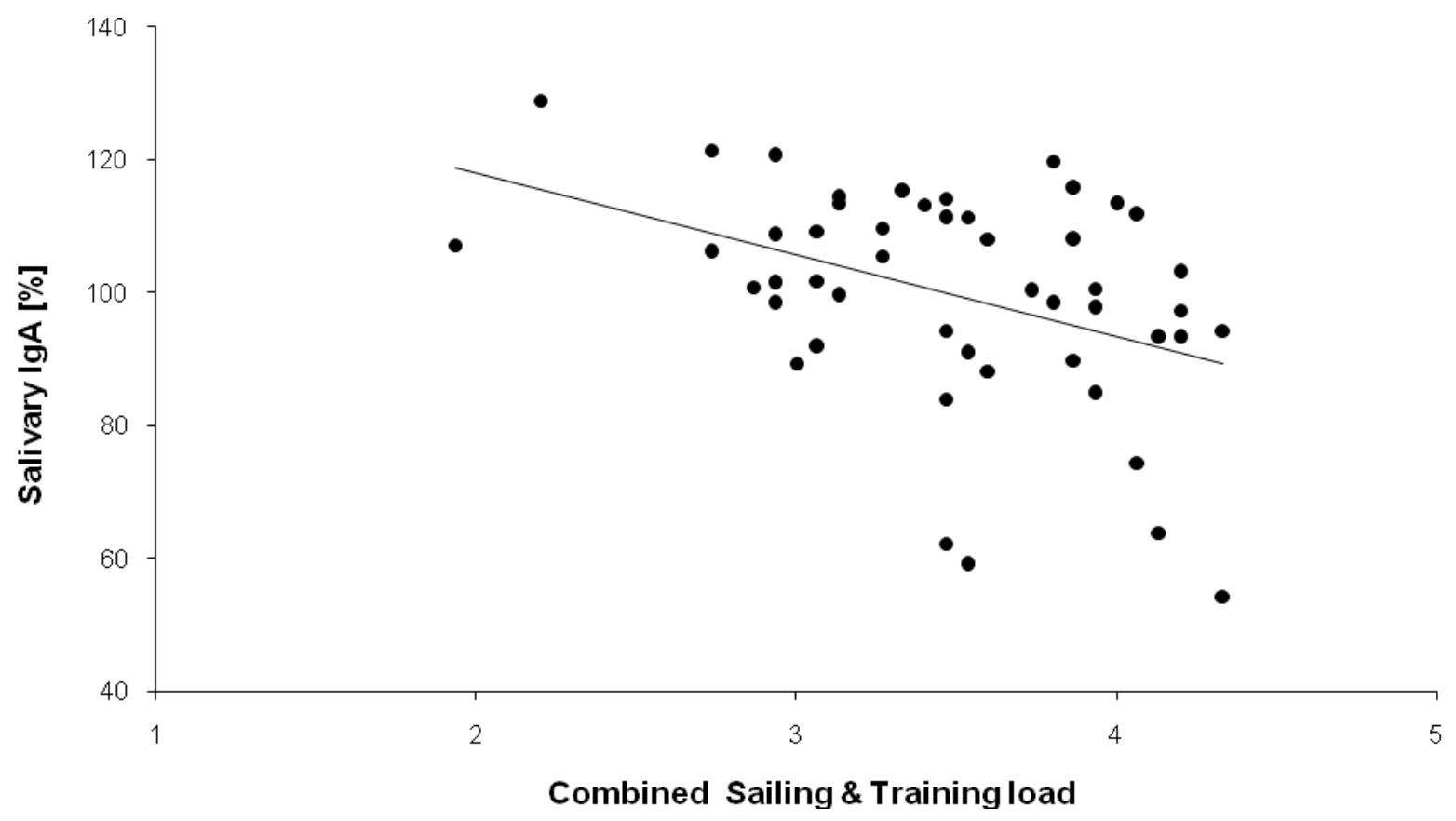

Figure 5: Scatter plot of the weekly Salivary $\operatorname{IgA}$, mean relative values, and the combined Sailing and Training load $\left(r=0.41, r^{2}=0.17, N=50, \mathrm{P}<0.005\right)$. 


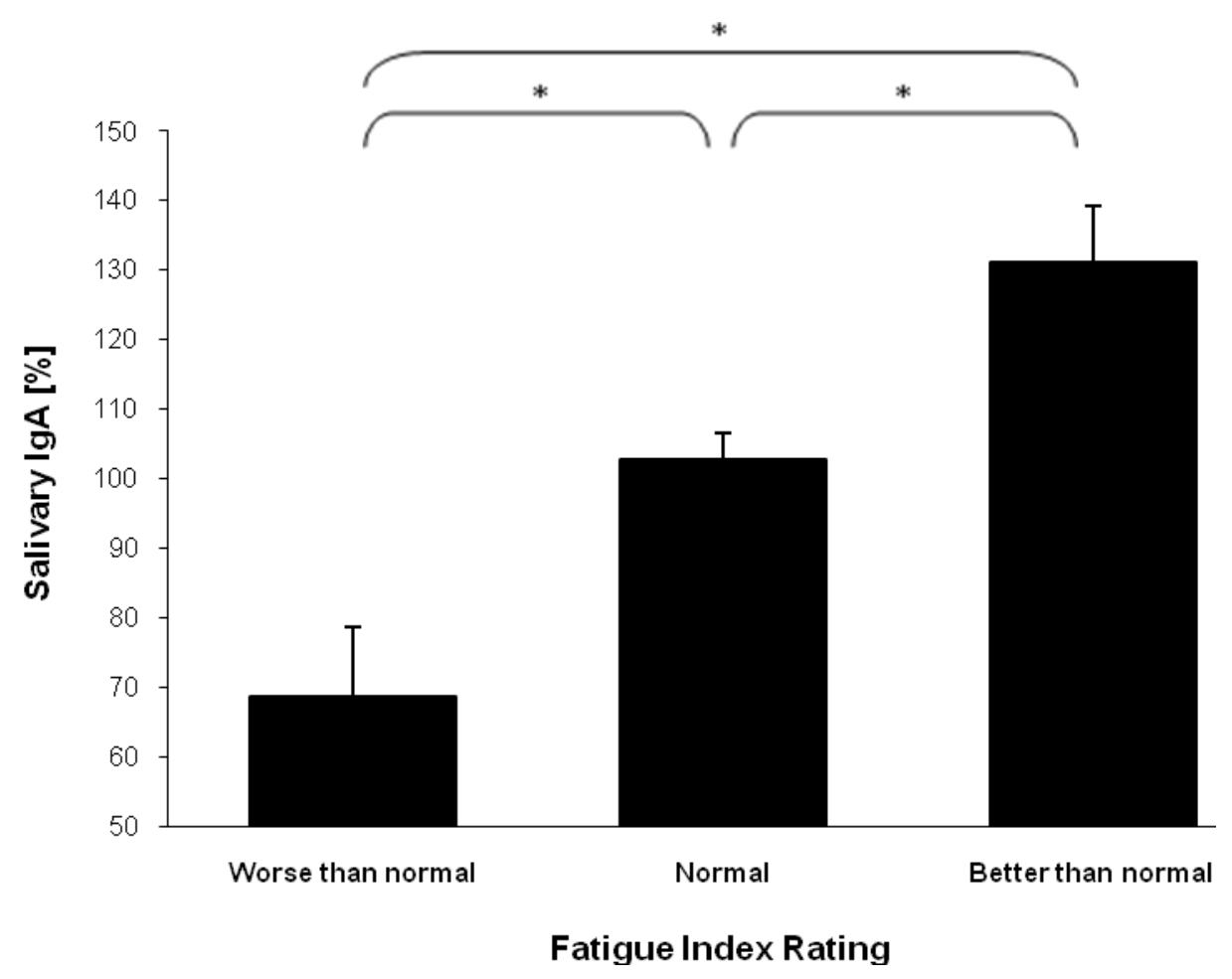

Figure 6: Salivary IgA concentration for different fatigue ratings. Data are Mean \pm SEM of 38 athletes, with each individual's relative S-IgA values averaged for each rating. * S-IgA for each rating significantly different to the other two $(N=38, \mathrm{P}<$ 0.005). 